\title{
Use of 3D printing for parent and patient education in repair of vascular ring and circumflex aorta
}

\author{
Robroy MacIver ${ }^{1}$, David Overman ${ }^{1}$, Brooke Moore ${ }^{1}$, Amanda Tenhoff ${ }^{2}$, Tinen Iles ${ }^{2}$, and \\ Paul Iazzio ${ }^{2}$ \\ ${ }^{1}$ Children's Hospitals and Clinics of Minnesota \\ ${ }^{2}$ University of Minnesota Twin Cities
}

January 21, 2021

\begin{abstract}
A clear understanding by the patient and family of airway pathology caused by vascular rings can be difficult to achieve. The pathology is three-dimensional in nature, and is a compilation of differing anatomic structures. Patient confusion can lead to misunderstandings regarding the nature of the operation, attendant risks, and the expected post-operative course. In this review we describe our use of 3D printing in the setting of circumflex aorta and double aortic arch to help guide a child and their family through the decision for treatment of what can be a difficult problem in both diagnosis and management. Our patient underwent two operations. First, a thoracoscopic division of a left ligamentum and atretic distal left aortic arch. While symptoms improved somewhat post-operatively, moderate dysphagia and dyspnea persisted. Based on further evaluation we concluded that the circumflex aorta was the etiology of the ongoing symptoms, so an aortic uncrossing procedure under circulatory arrest was recommended and performed. We used a 3D model in the education of the patient and family prior to the second surgery and greatly enhanced their understanding of why a repeat operation was required to address an additional component of this complex vascular ring. The use of 3D printing in preoperative discussions facilitated better understanding of complex three dimensional anatomy for the patient and patient's family. In addition, this communication strategy helped frame expectations regarding the post-operative course and convalescence.
\end{abstract}

\section{Introduction}

Vascular rings are comprised of multiple structures that together cause external compression on the airway and esophagus. Discussion and education regarding repair of simple rings such as division of a ligamentum arteriosum or double aortic arch is usually straight forward. With more complex anatomy, however, the goal of the operation can become lost in a description of terms and anatomical nuances that are difficult to communicate effectively to the patient and their family. Three dimensional (3D) printing can be a useful adjunct in the education of patients and their family prior to surgery.

We describe our treatment of a 9 year old female with right side dominant double aortic arch, with circumflex aorta. 3D printing was used in the description of the circumflex aorta and decision to pursue surgical treatment. Although a double aortic arch with an atretic limb is relatively common in the setting of vascular rings, a circumflex aorta is a rare congenital abnormality that can confound the proper diagnosis and can result in airway or esophageal compression.

\section{Materials and Methods}

Our patient initially presented at 7 years of age with shortness of breath with exertion and noisy breathing at rest. Her exertional symptoms were progressive, and she experienced prolonged recovery from upper respiratory infections. Subsequently she was no longer getting relief from bronchodilators that she had used 
up to that point. A chest x-ray revealed a right sided aortic arch. A computerized tomography (CT) scan demonstrated a double aortic arch with atretic distal left arch with no Kommerell's diverticulum. Bronchoscopy was performed which revealed $40 \%$ narrowing at the mid-trachea. Echocardiography examination provided a normal intra cardiac anatomy. Thoracoscopic division of the atretic left arch and left ligamentum was performed without incident and the patient recovered uneventfully.

Two years later the patient continued to have residual symptoms of shortness of breath with any exercise, including climbing a single flight of stairs. Her breathing at rest remained noisy, and she became breathless with talking for extended periods of time. Treatment with albuterol and fluticasone inhalers did not alleviate symptoms. An exercise study was performed to better delineate the mechanism of her symptoms which was read as consistent with mild obstruction. Both during her exercise testing and when performing spirometry at rest she elicited normal inspiratory loops, however her expiratory loop was flattened suggesting dynamic airway obstruction (Figure 1). Bronchoscopy was repeated which revealed normal vocal cord mobility, but compression at the lateral rightward aspect of the trachea (Figure 2). A CT scan was repeated to better delineate her anatomy.

Her case was discussed in our multidisciplinary congenital cardiac/thoracic conference where her repeat CT scan was reviewed. A rightward to leftward course of her aorta above the level of the carina was consistent with the diagnosis of circumflex aorta.

A discussion with the family over the phone where a description of the relevant anatomy and plan for treatment. Optimal understanding of the anatomy and its surgical solution was difficult to achieve. A $3 \mathrm{D}$ model of her anatomy using blood and air volumes was made using Materialise@ Mimics and 3-matic software (Leuven Belgium) and was printed on a an Ultimaker $(3)$ Extended printer using polylactic acid (PLA) filament. Figure 3 shows the resultant 3D model, created from her CT scan images. The postprocedural model was used preoperatively for the second operation to help describe the symptoms and the complex operation needed to provide relief of external compression of the trachea (1). This 3D model made conversation from this point on in the patient's care clear in both objectives and planning for outcomes.

\section{Results}

The patient underwent an uncrossing procedure under circulatory arrest. This ultimately resulted in dramatic relief of tension over the distal trachea visible upon intraoperative inspection. Intraoperative bronchoscopy revealed decrease in airway compression. Aortopexy was entertained as an adjunctive procedure, but did not appear to change the patency of her airway so was therefore not performed. We do not routinely perform a posterior tracheopexy in this setting. Her convalescence was notable for hypertension which was treated with oral agents. Post-operative echocardiography revealed no aortic arch obstruction and normal ventricular function. She experienced gradual improvement in symptoms as an outpatient. At her 6 month follow-up visit with her pulmonologist she was no longer breathless when speaking, her breathing was no longer noisy, and her shortness of breath with exertion had decreased. Follow-up spirometry was unchanged when compared to pre-operative measures. We provided the patient with the 3D model post operatively.

\section{Discussion}

This case illustrates how 3D printing can improve patient education and amplify understanding by clearly displaying all relevant anatomy in one modality. It was difficult for this patient and her family to fully understand the three dimensional anatomy of a circumflex aorta with verbal descriptions alone.

Originally discussed in a case report by Paul in 1948, Circumflex aorta was further described by the physician Raymond Heim de Balsac in 1960 with correspondence added by the pathologist Jessie Edwards $(2,3)$. Diagnoses depended at that time on combining multiple indirect modalities such as an esophagrams.

Circumflex aorta can occur in the setting of either a left or right aortic arch. The diagnosis requires the aortic transition in sidedness occur above the level of the carina. An operation to correct circumflex aorta was first described by Planche and LaCour-Gayet in 1984 (4). Subsequently a descriptive account using circulatory arrest was described by Russell et al (1). A clear understanding of the multiple structures manipulated to 
achieve symptomatic success is necessary. Key elements of a successful operation are avoidance of recurrent laryngeal nerve injury, lymphatic disruption leading to chylothorax, and vascular injury and hemorrhage during the division and translocation of the aorta.

Although rare, presentation is usually that of a child who has persistent symptoms after initial treatment of division of ligamentum arteriosum with or without an atretic distal arch. We would recommend a staged treatment pathway, as most patients will have effective relief of pathology by division of the ligamentum and atretic distal arch, if present. The aortic uncrossing procedure is reserved for those with persistent symptoms and evidence of ongoing airway impingement after initial repair. Postoperative follow up and reinvestigation are necessary to rule out continued anatomical causes of tracheomalacia and symptomatic compression in the setting of continued symptoms after division of vascular rings. The ubiquity of CT scanners makes re-investigation relatively simple.

3D printing has proved to be a useful adjunct to anatomical imaging. Although it may be considered redundant to 2D depictions of computer generated 3D constructs for surgical planning, its use in patient education can be quite impactful. In our case 3D modeling helped gain the trust and understanding of a family that had already gone through one operation, and was faced with a much larger operation with a higher risk of complications. Appropriately, there were doubts on the validity of the data presented through traditional methods such as 2D images and description of physiologic tests. Alleviating these doubts was aided with the use of a 3D model.

\section{Conclusions}

3D printing can be used as a useful adjunct for patient education prior to surgery in the setting of vascular rings in children.

Figure 1. Flow volume loop (exercise testing and at rest) after initial surgery releasing her vascular ring. There was a normal inspiratory loop, however her expiratory loop was flattened suggesting dynamic airway obstruction

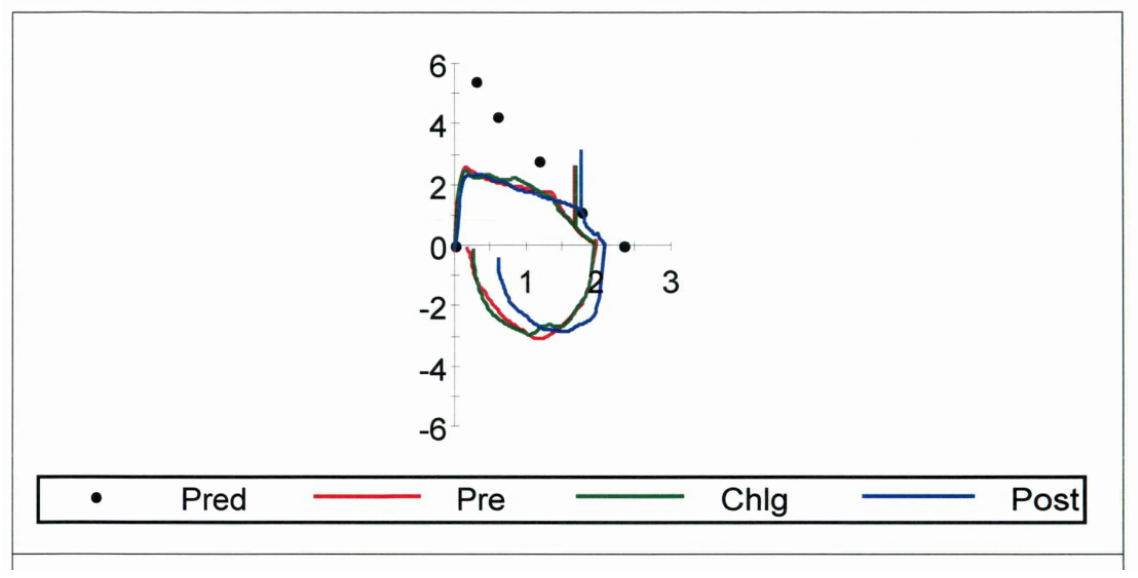

Figure 2. Bronchoscopy image performed after initial surgery revealing normal vocal cord mobility and compression at the lateral rightward aspect of the trachea 


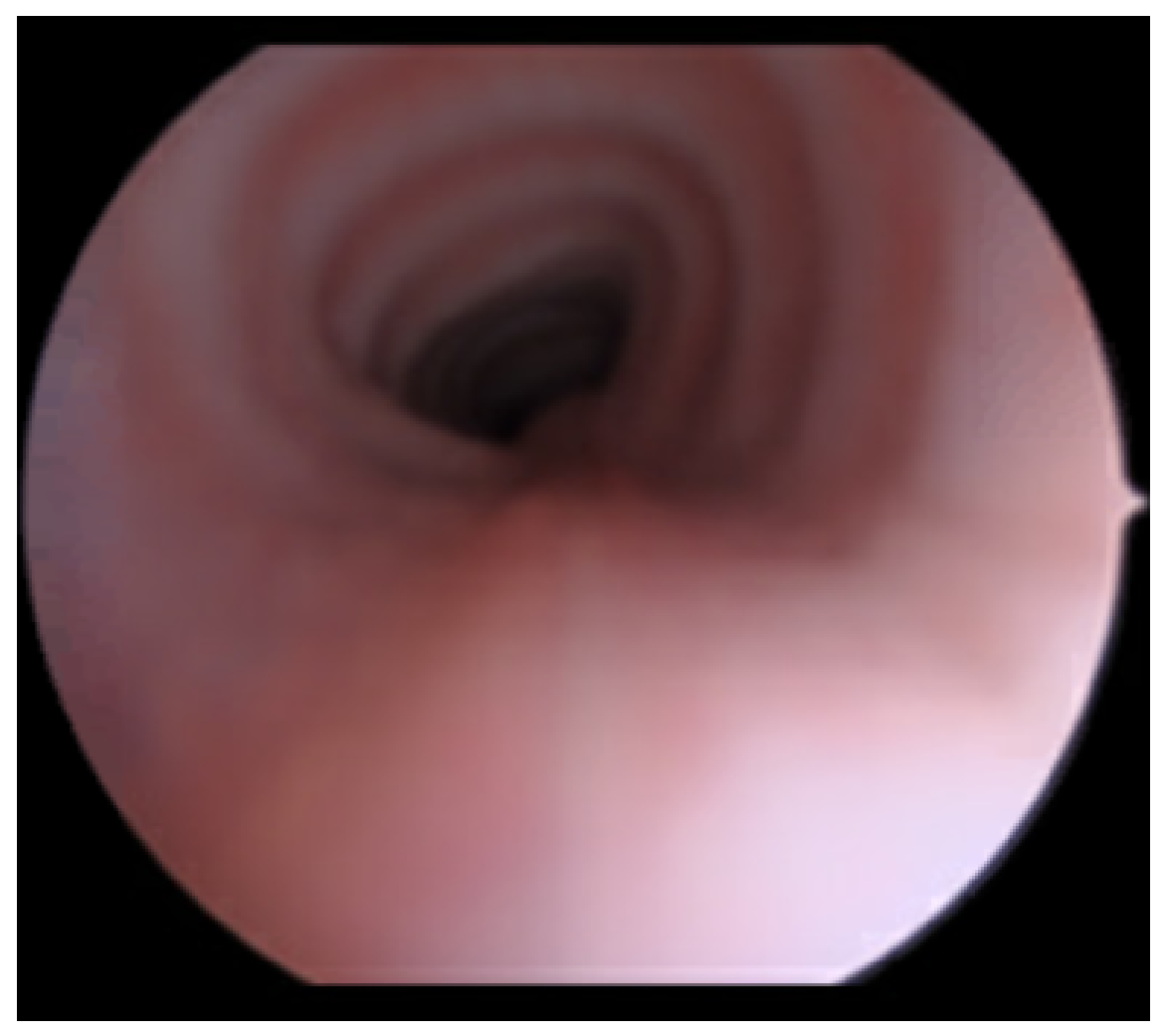

Figure 3. 3D model of the circumflex aorta generated from CT images and used for educating the child and her family.

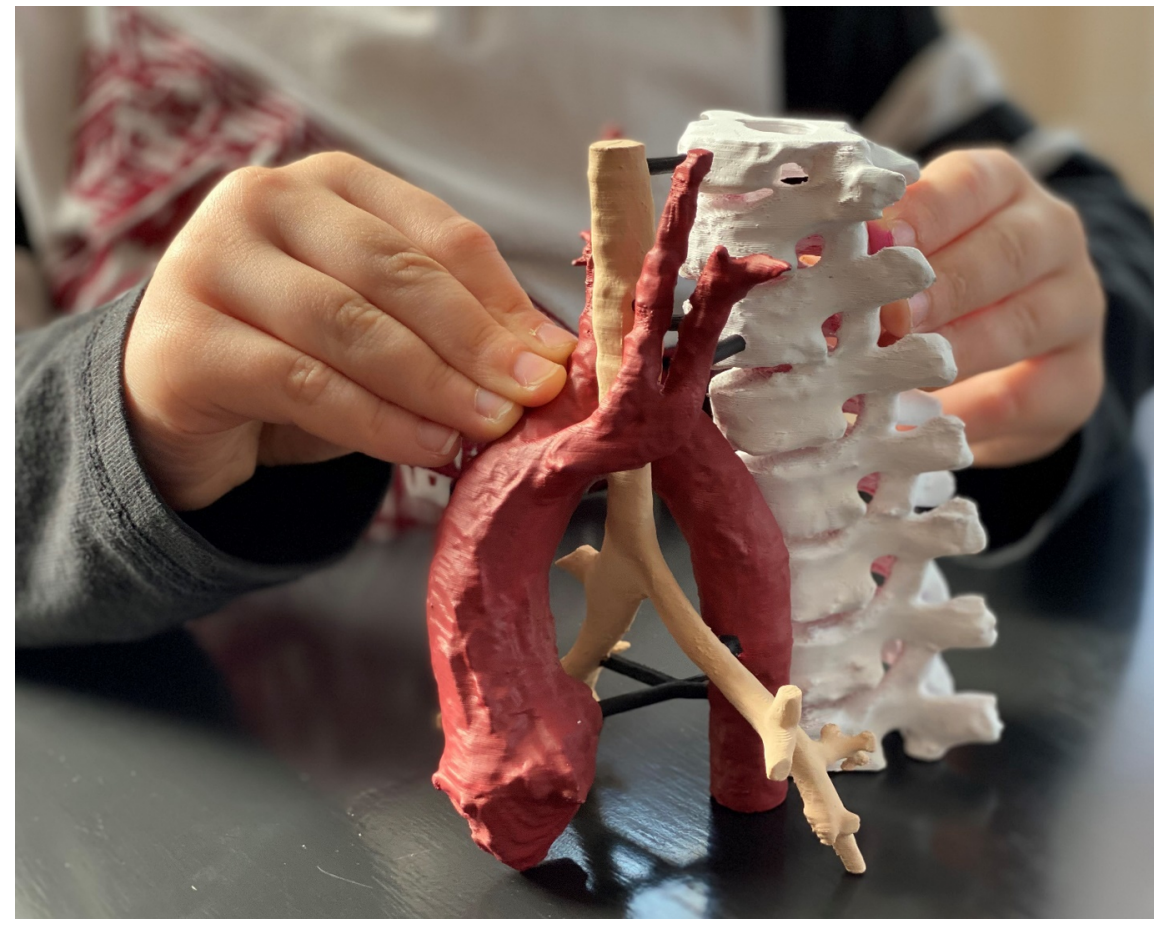




\section{References}

1. Russell HM, Rastatter JC, Backer CL. The Aortic Uncrossing Procedure for Circumflex Aorta. Operative Techniques in Thoracic and Cardiovascular Surgery. 2013;18(1):15-31.

2. Paul RN. A New Anomaly of the aorta; left aortic arch with right descending aorta. The Journal of Pediatrics. 1948;32:(119-29.

3. Balsac RH. "Left aortic arch (posterior or circumflex type) with right descending aorta". The American Journal of Cardiology. 1960;5(4):546-550.

4. Planche C, Lacour-Gayet F. "Aortic uncrossing for compressive circumflex aorta: 3 cases". Presse Med. 1984;13:1331-1332. (article in French) 